\title{
Coding Scheme for the Transmission of Satellite Imagery
}

\author{
Francesc Aulí-Llinàs ${ }^{\dagger}$, Michael W. Marcellin ${ }^{\ddagger}$, Victor Sanchez ${ }^{\S}$, \\ Joan Serra-Sagristà ${ }^{\dagger}$, Joan Bartrina-Rapesta ${ }^{\dagger}$, and Ian Blanes ${ }^{\dagger}$ \\ ${ }^{\dagger}$ Dep. of Information and Communications Engineering, Universitat Autònoma de Barcelona, Spain \\ ${ }^{\ddagger}$ Dep. of Electrical and Computer Engineering, University of Arizona, USA \\ $\S$ Dep. of Computer Science, The University of Warwick, United Kingdom
}

\begin{abstract}
The coding and transmission of the massive datasets captured by Earth Observation (EO) satellites is a critical issue in current missions. The conventional approach is to use compression on board the satellite to reduce the size of the captured images. This strategy exploits spatial and/or spectral redundancy to achieve compression. Another type of redundancy found in such data is the temporal redundancy between images of the same area that are captured at different instants of time. This type of redundancy is commonly not exploited because the required data and computing power are not available on board the satellite. This paper introduces a coding scheme for EO satellites able to exploit this redundancy. Contrary to traditional approaches, the proposed scheme employs both the downlink and the uplink of the satellite. Its main insight is to compute and code the temporal redundancy on the ground and transmit it to the satellite via the uplink. The satellite then uses this information to compress more efficiently the captured image. Experimental results for Landsat 8 images indicate that the proposed dual link image coding scheme can achieve higher coding performance than traditional systems for both lossless and lossy regimes.
\end{abstract}

\section{INTRODUCTION}

Since the launch of Sputnik 1 in 1957, the number and diversity of satellites orbiting the Earth has not stopped growing. Earth Observation (EO) satellites are a particular type devoted to the observation and monitoring of the surface of the Earth and its atmosphere. Their data are employed in applications to manage natural resources, forecast the weather, or monitor the climate change, among others. Commonly, EO satellites are situated in polar orbits, revolving around the Earth from the North to the South Pole. Except for the poles, these satellites cover different areas of the globe in each revolution due to the Earth's rotational movement. The most effective stations to download their data are located near the poles. These stations are in contact with the satellite in each revolution, though the transmission time is limited to a few minutes per orbit.

The imaging sensor of an EO satellite acquires huge amounts of data. The Landsat 8 satellite, for instance, carries two sensors that capture 11 spectral bands with a spatial resolution of up to 15 meters and a bit-depth resolution of up to 16 bits per sample (bps). The acquired data are downloaded to the ground station for further processing 
and distribution. Yet, it is common that satellite sensors can capture more data than the available downloading capacity. Often, this forces the acquired data to be trimmed down, which may negatively affect applications and users.

Satellites have two channels of communication: the downlink and the uplink. The downlink is employed to transmit the data acquired by the satellite to the ground station, whereas the uplink is employed to transmit commands and ancillary information from the ground station to the satellite. In EO satellites, the downlink has a much higher capacity than the uplink, and the particular configuration changes depending on the necessities of the sensors. The communication system has full duplex capacity, i.e., transmission can occur in both directions simultaneously. Advances in electronics and telecommunications have increased the capacity of the satellite channels to a few hundreds of megabits per second (Mbps) in the most modern satellites. Yet, each new EO satellite is equipped with sensors that can capture with more detail the surface, or the atmosphere, of our planet. This trend, which has been observed for the last two decades, together with the inability to provide significant additional improvements in download capacities, pulls down the acquisition capabilities of EO satellites.

The current approach to download the datasets captured by the satellites is the following. Once (or while) the image is acquired at the satellite, it is compressed using a coding system. Compression helps to reduce the size of the data. Both lossless and lossy compression regimes have been considered: the former allows perfect reconstruction while achieving low compression ratios; the latter improves the compression ratio at the expense of introducing distortion. Once the data are compressed -either with or without loss- to a size that fits the bit budget of the downlink, it is (stored and) transmitted during the time in which the ground station(s) are in contact with the satellite. Among others, standards and coding systems devised for the coding of satellite images using lossless and lossy regimes are respectively described in [1]-[6] and [2], [5], [7], [8].

Most techniques for the coding of satellite imagery either exploit the spatial or the spectral redundancy of the images or both. Nonetheless, there exists another type of redundancy that is usually not taken advantage of. Let us explain further. As previously described, EO satellites with polar orbits revolve around the Earth from the pole to pole. The Earth's rotation causes the satellite to capture a different area of the planet in each orbit but, after some number of orbits, the satellite is situated at the starting point again, scanning areas that were already captured. This is called the repeat cycle. The Landsat 8 satellite, for instance, has a repeat cycle of 16 days. As can be observed in Fig. 1, the images captured of the same area on different dates are very similar or, otherwise stated, they have high periodic temporal redundancy.

Current coding systems employed in satellites do not consider the temporal redundancy. In order to do so, different temporal samples of the same location would need to be available, which may not be possible. The Landsat 8 satellite may capture 650 images every day with a raw size of more than $300 \mathrm{MB}$ each. As the acquired data between repeat cycles is about $3 \mathrm{~TB}$, a mass memory of at least this size would be needed so that just two temporal samples of the same location would be available on board the 


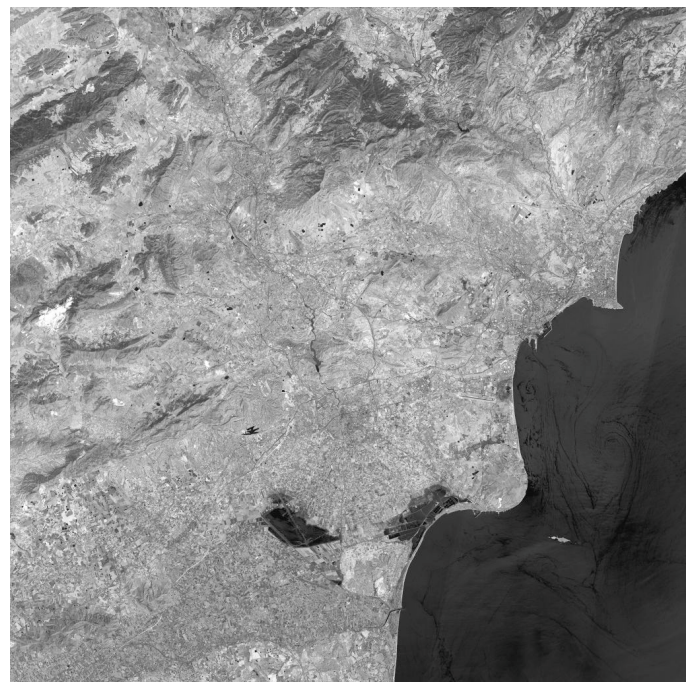

(a)

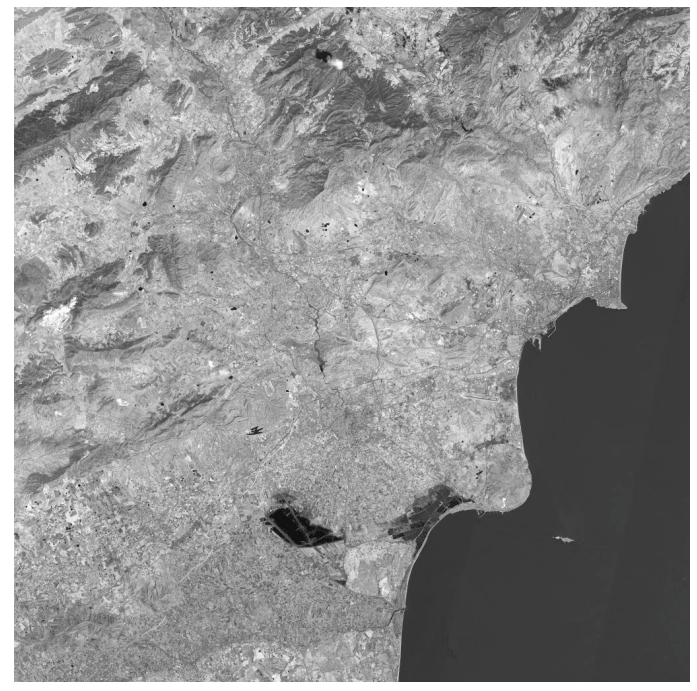

(b)

Fig. 1: Spectral band 6 of an image captured by the Landsat 8 over a region of Alicante (Spain) on (a) $7^{\text {th }}$ June and (b) $23^{\text {rd }}$ June of 2015.

satellite. In addition, properly exploiting such redundancy requires that temporal samples be co-registered, so that variations in satellite position and direction between consecutive repeat cycles are canceled. This is a computationally-demanding task that is performed on the ground before science data are disseminated. Hence, high requirements of mass memory and computational resources prevent a straightforward exploitation of temporal redundancy on the satellite.

The purpose of the research reported here is to enhance the ability of EO satellites to download the data acquired by their imaging sensors. To this end, our solution consists of exploiting the large computational power of the ground station and the capacity of the satellite's uplink, which is idle during most of the transmission time. The idea is that the ground station can use already-stored data to generate an estimate image. This estimate is then (coded and) transmitted via the uplink channel, allowing the satellite to benefit from the temporal redundancy during the compression process of the last image acquired. This coding scheme employs both the downlink and the uplink, so we refer to it as dual link image coding. The work described in this paper explores the performance that the dual link image coding scheme can achieve with Landsat 8 images. Some preliminary experimental results suggest that it may help to reduce the amount of data transmitted downwards significantly while transmitting only small amounts of data via the uplink.

The rest of the paper is organized as follows. Section II formulates the problem and describes the proposed approach. Section III presents experimental results. The last section concludes the paper with a brief summary. 


\section{DUAL LINK IMAGE CODING SCHEME}

\section{A. Traditional approach}

Let $X=\left\{X_{i}\right\}, 0 \leq i<N$, denote the image acquired by the satellite's sensor, with $N$ being the total number of samples in the image. Let $\mathcal{B}^{\downarrow}=\mathcal{T} \cdot \mathcal{C}^{\downarrow}$ denote the total bit budget of the downlink, with $\mathcal{T}$ and $\mathcal{C}^{\downarrow}$ being the time that the satellite is in contact with the ground station and the channel capacity of the downlink, respectively. The bit-depth resolution of the sensor is denoted by $D_{X}$. The size of the raw data is $S_{X}=N \cdot D_{X}$, whereas their order-zero entropy is referred to as $H(X)$. When $S_{X}>\mathcal{B}^{\downarrow}$, traditional approaches code the data to reduce their size before transmitting them down. For simplicity, in the following discussion we assume that a lossless compression system in the satellite produces a codestream whose length is that of the data's entropy [9]. The samples recovered at the ground station are referred to as $\widehat{X}=\left\{\widehat{X}_{i}\right\}$. When lossless compression is not attainable (i.e., when $H(X)>\mathcal{B}^{\downarrow}$ ), the coding process (transforms and) quantizes the data. The quantization procedure uses a step size, denoted by $\Delta_{\widehat{X}}$, that is commonly selected as the smallest that respects the bit budget (i.e., the smallest $\Delta_{\widehat{X}}$ that yields $H(\widehat{X}) \leq \mathcal{B}^{\downarrow}$ ). The (transformed) samples recovered at the ground station are computed as

$$
\widehat{X}_{i}=\left(\left\lfloor\frac{X_{i}}{\Delta_{\widehat{X}}}\right\rfloor+\delta\right) \cdot \Delta_{\widehat{X}},
$$

with $\lfloor\cdot\rfloor$ denoting the floor operation. When $\Delta_{\widehat{X}}=2^{D_{X}-D_{\widehat{X}}}$, the quantization procedure is equivalent to discarding the $D_{X}-D_{\widehat{X}}$ least significant bits of the (transformed) samples. Other values of $\Delta_{\widehat{X}}$ may help to adjust more finely $H(\widehat{X})$ to fit $\mathcal{B}^{\downarrow}$. The division and floor operation in (1) are carried out on the satellite, whereas the remaining operations are carried out at the station. $\delta \in[0,1)$ is the reconstruction factor employed to recover the samples. Typically, $\delta=0.5$.

When mean squared error (MSE) is the quality metric employed, the image distortion is computed as $D=\frac{1}{N} \sum_{i}\left(X_{i}-\widehat{X}_{i}\right)^{2}$. The objective of traditional lossy coding schemes is to minimize the distortion while respecting the total bit budget of the downlink, i.e.,

$$
\min D \quad \text { s.t. } \quad H(\widehat{X}) \leq \mathcal{B}^{\downarrow}
$$

\section{B. Proposed approach}

Contrary to traditional approaches, the dual link image coding scheme utilizes both the downlink and the uplink. The main idea is that some information, say $s$, is computed in the ground station and transmitted to the satellite via the uplink. The computation of $s$ may employ huge amounts of data and/or complex algorithms since computational power is not constrained on the ground. Once transmitted, the satellite uses $s$ to code $X$. If $s$ is correlated with $X$, then $H(X \mid s)<H(X)$, so the amount of information 


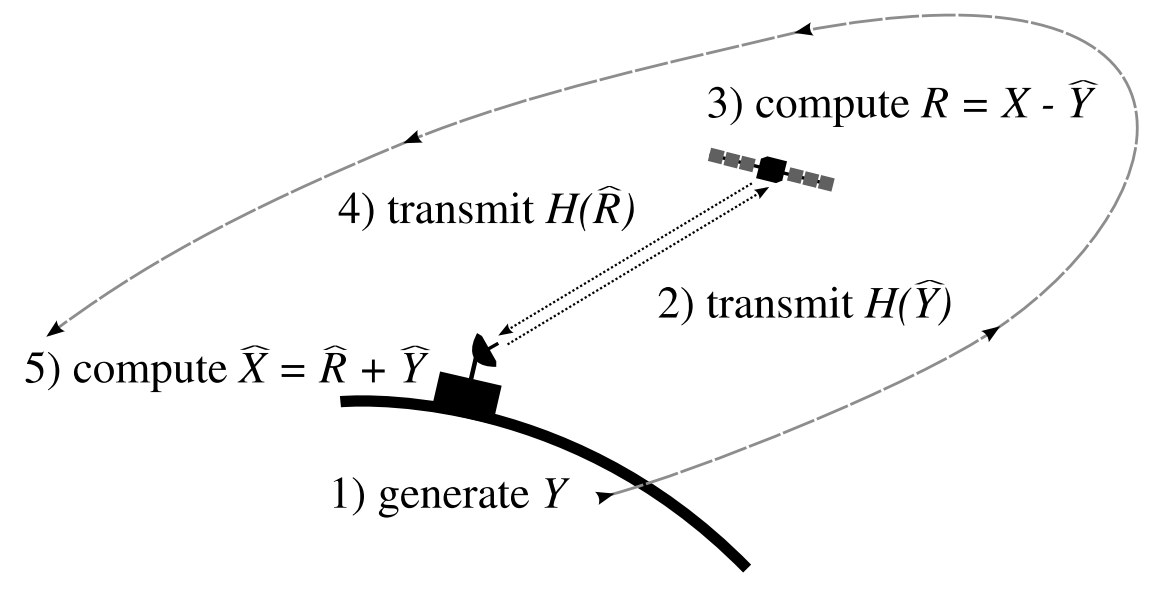

Fig. 2: Overview of the dual link image coding scheme.

that is transmitted downwards is reduced, at most, by their mutual information (i.e., by $I(X ; s))$.

Key to achieving high coding efficiency is to obtain an $s$ sufficiently similar to $X$. As previously described, EO satellites cover regions of the Earth in repeat cycles, so the images captured in the previous cycles are likely to be similar to the current. In this paper we explore the particular case in which $s$ is exactly that image captured in the previous cycle, which is called the estimate and is referred to as $Y=\left\{Y_{i}\right\}$. The total bit budget of the uplink is denoted by $\mathcal{B}^{\uparrow}=\mathcal{T} \cdot \mathcal{C}^{\uparrow}$, with $\mathcal{C}^{\uparrow}$ being its channel capacity. The bit-depth of $Y$ is the same as that of $X$ (i.e., $D_{Y}=D_{X}$ ). $Y$ needs to be coded when $S_{Y}=N \cdot D_{Y}>\mathcal{B}^{\uparrow}$. Again, the coding process employs (transformation and) quantization. The (transformed) samples recovered on the satellite are computed like in (1), i.e.,

$$
\widehat{Y}_{i}=\left(\left\lfloor\frac{Y_{i}}{\Delta_{\widehat{Y}}}\right\rfloor+\delta\right) \cdot \Delta_{\widehat{Y}},
$$

with $\widehat{Y}_{i}$ and $\Delta_{\widehat{Y}}$ denoting the recovered samples of the estimate and the step size, respectively. Note that in (3) the division and floor operation are carried out in the ground station, whereas the other operations are performed on board. Any $\Delta_{\widehat{Y}}$ that satisfies $H(\widehat{Y}) \leq \mathcal{B}^{\uparrow}$ may be chosen.

One simple approach is for the satellite to compute the residual image, referred to as $R$, via $R_{i}=X_{i}-\widehat{Y}_{i}$. Of course, better performing (but more complex) methods could be employed. The bit-depth of the residual is $D_{R}=D_{X}+1$. If $H(R)>\mathcal{B}^{\downarrow}$, then quantization is needed to transmit $R$ to the ground station. The coding process (transforms and) quantizes $R$ employing a step size $\Delta_{\widehat{R}}$ so that $H(\widehat{R}) \leq \mathcal{B}^{\downarrow}$. Once $\widehat{R}$ is at the ground station, the image is recovered employing $\widehat{R}$ and $\widehat{Y}$. With some abuse of notation, the samples recovered at the ground station through the dual link coding scheme are also referred to as $\widehat{X}$. They are computed as $\widehat{X}_{i}=\widehat{Y}_{i}+\widehat{R}_{i}$. Fig. 2 summarizes the steps of 


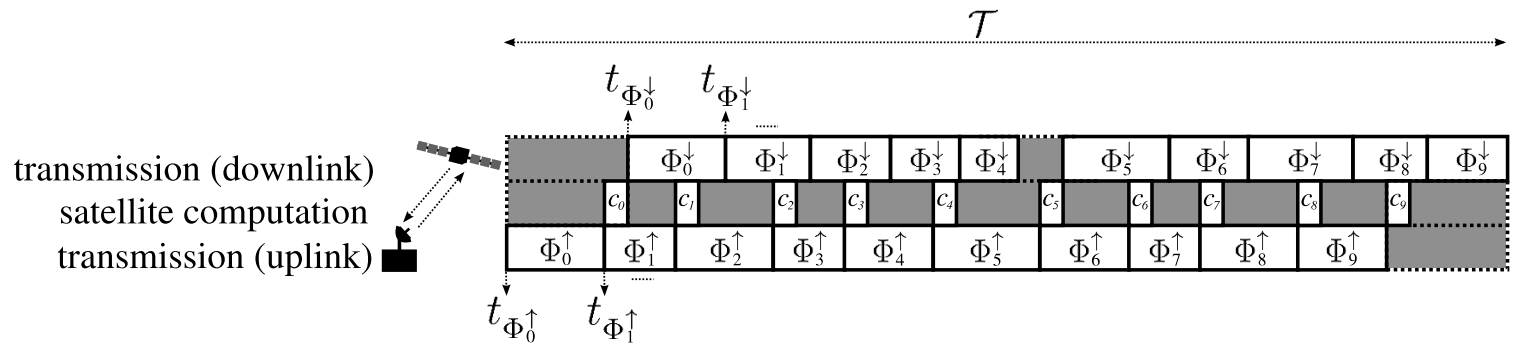

Fig. 3: Illustration of the synchronization needed to transmit and process packets of data.

the dual link image coding scheme.

Using MSE as the quality metric, the objective of the proposed scheme is to minimize the distortion while respecting both the total bit budget of the uplink and the downlink, i.e.,

$$
\min D \quad \text { s.t. } H(\widehat{Y}) \leq \mathcal{B}^{\uparrow} \text { and } H(\widehat{R}) \leq \mathcal{B}^{\downarrow}
$$

\section{Synchronization aspects}

The application of the dual link image coding scheme needs to consider the synchronization between the ground station and the satellite. In order to compute $R_{i}$, the satellite needs $\widehat{Y}_{i}$, so some kind of synchronization is required. Let us assume that $\widehat{Y}$ is transmitted in $J$ packets of data. Each packet contains $M=N / J$ samples. The packets are denoted by $\Phi_{j}^{\uparrow}, 0 \leq j<J$, and contain a set of (transformed and) quantized samples, more precisely, $\Phi_{j}^{\uparrow}=\left\{\widehat{Y}_{j \cdot M}, \ldots, \widehat{Y}_{((j+1) \cdot M)-1}\right\}$. Once $\Phi_{j}^{\uparrow}$ is uploaded, the satellite decodes it, computes the corresponding residuals, and codes them in a packet referred to as $\Phi_{j}^{\downarrow}$ that contains $\Phi_{j}^{\downarrow}=\left\{\widehat{R}_{j \cdot M}, \ldots, \widehat{R}_{((j+1) \cdot M)-1}\right\}$. The entropy of these packets is denoted by $H\left(\Phi_{j}^{\uparrow}\right)$ and $H\left(\Phi_{j}^{\downarrow}\right)$. The time spent to transmit them is $\mathcal{T}_{\Phi_{j}^{\uparrow}}=H\left(\Phi_{j}^{\uparrow}\right) / \mathcal{C}^{\uparrow}$ and $\mathcal{T}_{\Phi_{j}^{\downarrow}}=H\left(\Phi_{j}^{\downarrow}\right) / \mathcal{C}^{\downarrow}$. As seen in Fig. 3, packets $\Phi_{j}^{\uparrow}$ are uploaded consecutively, with $t_{\Phi_{j}^{\uparrow}}=\sum_{k=0}^{j-1} \mathcal{T}_{\Phi_{k}^{\uparrow}}$ being the instant at which packet $\Phi_{j}^{\uparrow}$ is beginning to be transmitted. The transmission of $\Phi_{j}^{\downarrow}$ must consider that the satellite needs some time to decode $\Phi_{j}^{\uparrow}$ and to compute the residuals. This computation time is referred to as $c_{j}$ for packet $j . t_{\Phi_{j}^{\downarrow}}$ is then computed as

$$
t_{\Phi_{j}^{\downarrow}}=\max \left(c_{j}+\sum_{k=0}^{j} \mathcal{T}_{\Phi_{k}^{\uparrow}}, t_{\Phi_{j-1}^{\downarrow}}+\mathcal{T}_{\Phi_{j-1}^{\downarrow}}\right),
$$

which corresponds to the earliest instant at which the downlink is not occupied and the packet is ready. For packets $\Phi_{0}^{\downarrow}$ and $\Phi_{5}^{\downarrow}$ depicted in Fig. 3, $t_{\Phi_{j}^{\downarrow}}$ is that instant in which packets $\Phi_{k}^{\uparrow}, 0 \leq k \leq j$ are already transmitted plus the time needed to compute 
$\Phi_{j}^{\downarrow}$, expressed in the left side of the $\max (\cdot)$ expression in (5). For the other packets of the figure, the earliest instant is when the previous packet (i.e., $\Phi_{j-1}^{\downarrow}$ ) has already been transmitted, which is expressed in the right part of the $\max (\cdot)$ expression. Note that (5) assumes that processing times $c_{j}$ are shorter than transmission times $\mathcal{T}_{\Phi_{i}^{\dagger}}$.

The synchronization needed between the ground station and the satellite reduces the effective bit budget of both channels since, as seen in Fig. 3, there can be periods in which one or both channels are idle. The effective bit budget of the uplink is determined as $\mathcal{B}^{\prime \uparrow}=\left(\sum_{j} \mathcal{T}_{\Phi_{j}^{\uparrow}}\right) \cdot \mathcal{C}^{\uparrow}$, whereas that of the downlink is determined as $\mathcal{B}^{\downarrow}=\left(\sum_{j} \mathcal{T}_{\Phi_{j}^{\downarrow}}\right) \cdot \mathcal{C}^{\downarrow}$. In practice, the reduction in the bit budgets is not significant because the transmission of the packets and their processing is mostly performed in pipeline, as depicted in Fig. 3.

Depending on the requirements of the scenario, the dual link image coding scheme can use more or less packets of data. In general, we envisage three levels of synchronization. The first is for samples (i.e., when $J=N$ ). Each sample is coded and transmitted independently from the others. This maximizes $\mathcal{B}^{\uparrow}$ and $\mathcal{B}^{\prime \downarrow}$. Nonetheless, it may penalize compression efficiency since each estimate/residual is coded independently. At the other extreme, the synchronization can be carried out at the level of the image (i.e., when $J=1)$. This may help to reduce $H\left(\Phi_{0}^{\uparrow}\right)$ and $H\left(\Phi_{0}^{\downarrow}\right)$ though it may halve the bit budget of the channels since $t_{\Phi_{0}^{\downarrow}}$ is significantly increased. When $0<J<N$, each packet may contain a reasonable number of estimates/residuals, achieving a tradeoff between the size of the packets and the bit budget of the channels. Many coding systems provide a division of image data into blocks or tiles that may naturally correspond to these packets of data.

We remark that the synchronization discussed above is necessary when the satellite transmits the image captured in the latest orbit. If the satellite had enough computational power, such synchronization might be avoided by delaying the transmission of the last image by one orbit, i.e., by sending up the estimate of the image acquired in the current orbit, and sending down the residual of the image captured in the previous orbit. In this case, the residual may be computed and coded during the entire orbit rather than during the time in which the satellite is in contact with the ground station.

\section{EXPERIMENTAL RESULTS}

The dual link image coding scheme is evaluated for coding the Landsat 8 image depicted in Fig. 1, which covers a region of Alicante (Spain), and another Landsat 8 image that covers a region of Cartagena (Spain). These images correspond to a portion of the WRS-2 path 199, row 33 and WRS-2 path 199, row 34 captured on $23^{\text {rd }}$ June, 2015 and $12^{\text {th }}$ February, 2014, respectively. Both images are $2048 \times 2048$ with a bit-depth resolution of 16 bits per sample and have 11 spectral components. They are provided by the U.S. Geological Survey [10]. As previously stated, the estimate image corresponds to the immediately previous capture from the same area. Since the Landsat 8 satellite has a repeat cycle of 16 days, the estimates were captured on $7^{\text {th }}$ June, 2015 and $27^{\text {th }}$ January, 2014, respectively for the "Alicante" and "Cartagena" images.

The first test evaluates lossless coding performance. Fig. 4 depicts the results obtained. The figure reports the minimum bit budget necessary to transmit the images with no 


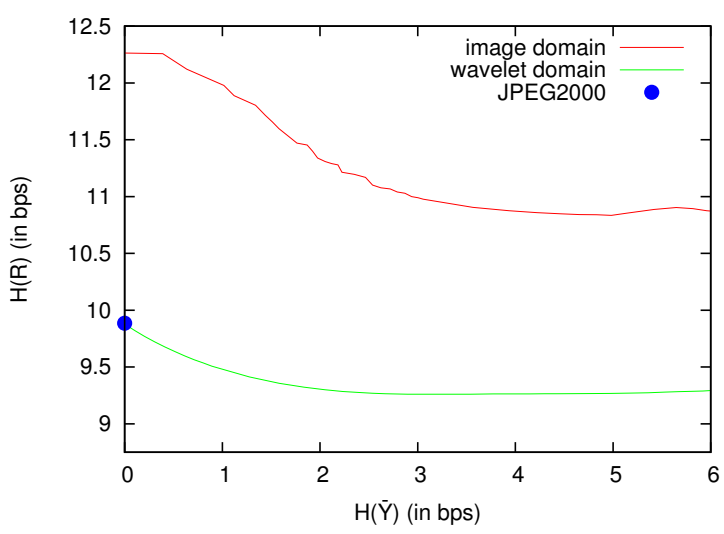

(a)

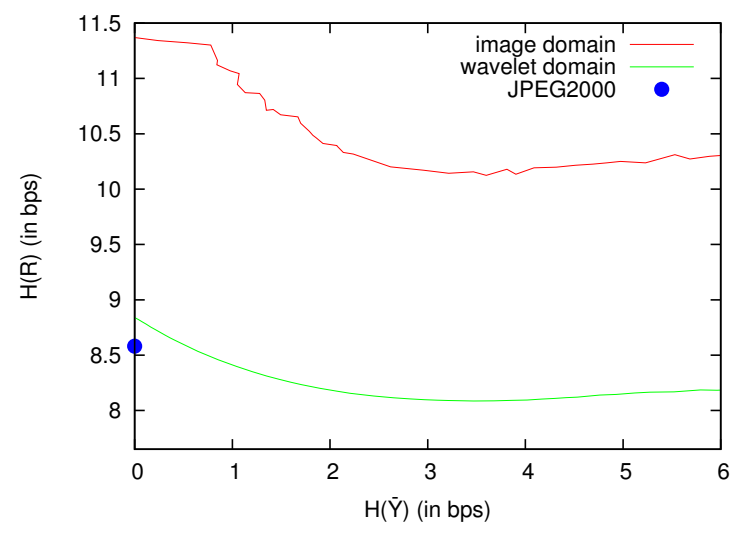

(b)

Fig. 4: Evaluation of the lossless coding performance of the dual link image coding scheme for the (a) "Alicante" and (b) "Cartagena" images.

losses, for both the uplink and the downlink. The horizontal axis is the entropy of the quantized estimates transmitted upwards (i.e., $H(\widehat{Y})$ ), whereas the vertical axis is the entropy of the residuals transmitted downwards (i.e., $H(R)$ ). Note that even though the estimates are quantized, the residuals are not quantized in order to achieve lossless compression. Both axes report the results in bits per sample (bps). The figure depicts the results achieved when the image samples are directly transmitted and when they are transformed via the reversible CDF 5/3 wavelet transform using 5 levels of decomposition. The wavelet transform is applied in the spatial domain only. When the image is transformed, quantization is applied to the wavelet coefficients of the estimate, but not of the residual. The results of Fig. 4 indicate that the use of the wavelet transform reduces the entropy of the signal by approximately $2.5 \mathrm{bps}$. Regardless of whether or not the wavelet transform is employed, transmission of the estimate increases the compression efficiency of the on board coding system. This is seen in the figure as the decreasing amount of data that is transmitted via the downlink as more data are transmitted upwards. The transmission of more than 3 bps via the uplink does not provide further gains in compression efficiency, and actually may decrease efficiency slightly.

Fig. 4 also provides the lossless compression performance achieved by JPEG2000 when no estimate is transmitted, corresponding to a traditional downlink compression system. The image is compressed employing 5 levels of reversible CDF 5/3 wavelet transform, $64 \times 64$ codeblocks, and a single quality layer codestream. The results are reported as a point along the left vertical axis of the figure corresponding to no data being transmitted upwards. For the "Alicante" image, the lossless compression rate of JPEG2000 is nearly the same as the entropy of the wavelet coefficients. This is mainly because JPEG2000 may not be efficient to compress some of these images [9]. For the "Cartagena" image, the lossless performance of JPEG2000 is somewhat better than the entropy of the wavelet coefficients. In both cases, the proposed dual link image coding 


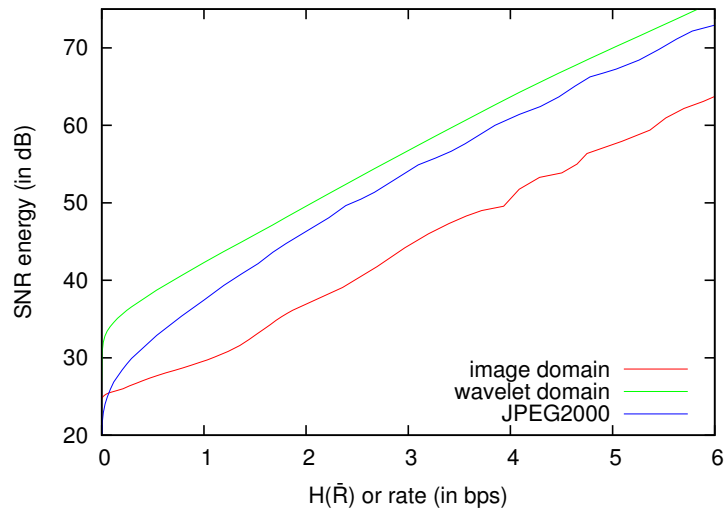

(a)

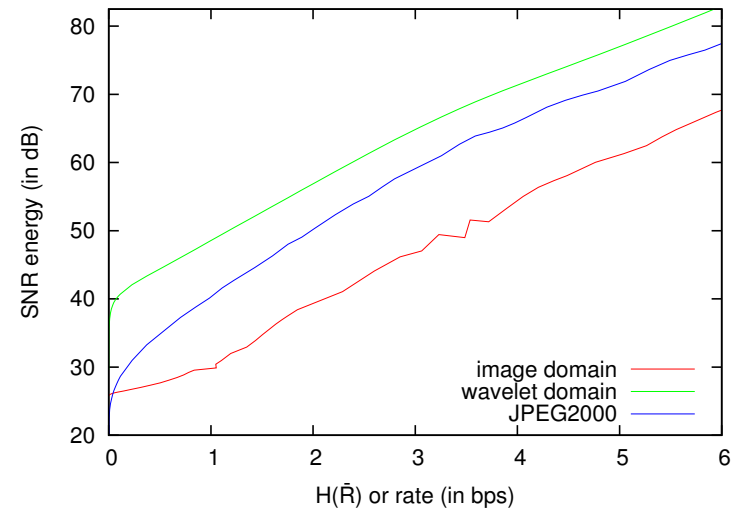

(b)

Fig. 5: Evaluation of the lossy coding performance of the dual link image coding scheme for the (a) "Alicante" with $H(\widehat{Y})=2$ bps and (b) "Cartagena" with $H(\widehat{Y})=1$ bps.

scheme reduces the amount of data needed to be transmitted through the downlink even when the bit budget of the uplink is very small compared to the downlink bit budget.

The second test evaluates lossy coding performance. To do so, the bit budget of the uplink is fixed to a specific rate of $\mathcal{B}^{\uparrow}=2$ bps for the "Alicante" image and $\mathcal{B}^{\uparrow}=1$ bps for the "Cartagena" image. Fig. 5 depicts the results obtained. The horizontal axis is the entropy of the quantized residuals, whereas the vertical axis is the quality of the image, reported as the Signal to Noise Ratio (SNR). Again, results for both the transmission of image samples and wavelet coefficients are reported. The wavelet transform employed in these experiments is the irreversible CDF 9/7 with 5 levels of decomposition. Results for the lossy mode of JPEG2000, which uses the same wavelet transform, are also reported in the figure. As before, the results of JPEG2000 correspond to the case where no estimate is employed, i.e., when $\mathcal{B}^{\uparrow}=0$. These results suggest that the dual link image coding scheme may help to increase the quality of the images significantly. When the images are wavelet transformed, the gains are of more than $10 \mathrm{~dB}$ at low rates as compared with those of JPEG2000. When the image is transmitted without transform, the quality of the image is much poorer than when it is wavelet transformed. Similar results are obtained with different capacities of the uplink (not shown in the figure).

As stated in the previous section, these experimental results evaluate the performance of the dual link image coding scheme via the order-zero entropy of the data. As indicated in [9], the results are indicative of the performance that can be achieved with practical coding systems. The use of the order-zero entropy of the data simplifies the synchronization between the ground station and the satellite because each sample is coded and transmitted independently from the others. In other words, $J=N$ so almost the maximum bit budget of both channels can be employed as previously described. 


\section{CONCLUSIONS}

Earth Observation satellites are a particular type of satellites devoted to the observation and monitoring of the Earth. They are equipped with high-resolution imaging sensors that acquire huge amounts of data. Often, the size of the datasets captured exceeds that of the downlink capacity, so they have to be trimmed down and/or compressed. In general, coding systems for satellite imagery compress the image on board and then transmit it down. Contrary to traditional approaches, this work introduces a coding scheme that uses both the downlink and the uplink to code and transmit the image. The main idea is to employ images of the same region that are available on the ground to produce an estimate of the image captured at the satellite. This estimate is transmitted via the uplink, which is otherwise idle during most of the transmission time. The redundancy between the estimate and the captured image is employed by the on-board coding system to compress more efficiently the captured image, increasing the quality of the transmitted image and/or allowing more images to be downloaded. Experimental results carried out with Landsat 8 images suggest that the dual link image coding scheme may help to reduce the entropy of the downloaded images significantly. For lossless regimes, the proposed scheme reduces the required rate by approximately 0.5 bps compared to that achieved by JPEG2000 operating in a traditional fashion. For lossy regimes, the proposed scheme can increase the quality of the images by more than $10 \mathrm{~dB}$, especially at low rates. Future work will explore practical coding systems in conjunction with the dual link image coding scheme.

\section{ACKNOWLEDGMENT}

This work has been partially supported by the Spanish Government (MINECO), by FEDER, and by the Catalan Government, under Grants RYC-2010-05671, TIN201571126-R, TIN2012-38102-C03-03, and 2014SGR-691.

\section{REFERENCES}

[1] Lossless Data Compression, Consultative Committee for Space Data Systems Std. CCSDS 121.0-B, May 1997. [Online]. Available: http://public.ccsds.org/publications/archive/121x0b2.pdf

[2] Image Data Compression, Consultative Committee for Space Data Systems Std. CCSDS 122.0-B, Nov. 2005. [Online]. Available: http://public.ccsds.org/publications/archive/122x0b1c3.pdf

[3] Lossless Multispectral \& Hyperspectral Image Compression, Consultative Committee for Space Data Systems Std. CCSDS 123.0-B, May 2012. [Online]. Available: http://public.ccsds.org/publications/archive/123x0b1ec1.pdf

[4] A. Abrardo, M. Barni, E. Magli, and F. Nencini, "Error-resilient and low-complexity onboard lossless compression of hyperspectral images by means of distributed source coding," IEEE Trans. Geosci. Remote Sens., vol. 48, no. 4, pp. 1892-1904, Apr. 2010.

[5] C. Kai-jen and J. Dill, "Lossless to lossy dual-tree BEZW compression for hyperspectral images," IEEE Trans. Geosci. Remote Sens., vol. 52, no. 9, pp. 5765-5770, Sep. 2014.

[6] M. Mamun, X. Jia, and M. Ryan, "Nonlinear elastic model for flexible prediction of remotely sensed multitemporal images," IEEE Geosci. Remote Sens. Lett., vol. 11, no. 5, pp. 1005-1009, May 2014.

[7] A. Kiely and M. Klimesh, “The ICER Progressive Wavelet Image Compressor," IPN Progress Report, Tech. Rep., Nov. 2003. [Online]. Available: http://ipnpr.jpl.nasa.gov/tmo/progress_report/42-155/155J.pdf

[8] Q. Du, N. Ly, and J. Fowler, "An operational approach to PCA+JPEG2000 compression of hyperspectral imagery," IEEE J. Sel. Topics Appl. Earth Observations Remote Sens., vol. 7, no. 6, pp. 2237-2245, Jun. 2014.

[9] F. Auli-Llinas, "Entropy-based evaluation of context models for wavelet-transformed images," IEEE Trans. Image Process., vol. 24, no. 1, pp. 57-67, Jan. 2015.

[10] U. G. Survey. (2015, Sep.) Earth explorer. [Online]. Available: http://earthexplorer.usgs.gov 